University of Wollongong

Research Online

Australian Institute for Innovative Materials -

Papers

Australian Institute for Innovative Materials

2000

Microscopic calculations of linear and quadratic optical response in model Langmuir-Blodgett multilayers

Marc in het Panhuis

University of Wollongong, panhuis@uow.edu.au

R W. Munn

UMIST

Follow this and additional works at: https://ro.uow.edu.au/aiimpapers

Part of the Engineering Commons, and the Physical Sciences and Mathematics Commons

Research Online is the open access institutional repository for the University of Wollongong. For further information contact the UOW Library: research-pubs@uow.edu.au 


\title{
Microscopic calculations of linear and quadratic optical response in model Langmuir-Blodgett multilayers
}

\author{
Abstract \\ Microscopic calculations are reported of linear and quadratic nonlinear optical response in \\ Langmuir-Blodgett films. The films are modeled as ordered layers of molecules that can tilt from the \\ vertical and have a higher polarizability and first hyperpolarizability in the head than in the tail. The local \\ electric field varies little after the first layer and negligibly after the second, which simplifies the analysis. \\ Tilted two-layer $Y$-type films can have quadratic susceptibility components as large as $X$ - or Z-type ones. \\ Overall the results provide a systematic account of the linear and nonlinear optical properties as a \\ function of molecular and filmstructure that should assist interpretation of experimental results.

\section{Keywords} \\ optical, response, langmuir, calculations, linear, quadratic, multilayers, model, microscopic, blodgett \\ Disciplines \\ Engineering | Physical Sciences and Mathematics

\section{Publication Details} \\ in het Panhuis, M. \& Munn, R. W. (2000). Microscopic calculations of linear and quadratic optical response \\ in model Langmuir-Blodgett multilayers. Journal of Chemical Physics, 112 (15), 6763-6773.
}




\section{AD| $\begin{aligned} & \text { The Journal of } \\ & \text { Chemical Physics }\end{aligned}$}

\section{Microscopic calculations of linear and quadratic optical response in model Langmuir-Blodgett multilayers}

M. in het Panhuis and R. W. Munn

Citation: The Journal of Chemical Physics 112, 6763 (2000); doi: 10.1063/1.481238

View online: http://dx.doi.org/10.1063/1.481238

View Table of Contents: http://scitation.aip.org/content/aip/journal/jcp/112/15?ver=pdfcov

Published by the AIP Publishing

\section{Articles you may be interested in}

Variation in surface plasmonic response due to the reorganization of Au nanoparticles in Langmuir-Blodgett film J. Appl. Phys. 116, 024316 (2014); 10.1063/1.4890034

Orange-red luminescence from Cu doped CdS nanophosphor prepared using mixed Langmuir-Blodgett multilayers

J. Chem. Phys. 128, 114703 (2008); 10.1063/1.2888930

Langmuir-Blodgett films of polyethylene

J. Appl. Phys. 92, 5977 (2002); 10.1063/1.1513201

Microscopic treatment of substrate effects on linear and quadratic optical response of model Langmuir-Blodgett multilayers

J. Chem. Phys. 113, 10685 (2000); 10.1063/1.1323725

Analysis of linear and quadratic optical response of mixed Langmuir-Blodgett films of stearic acid and 5-CT J. Chem. Phys. 113, 10691 (2000); 10.1063/1.1323724

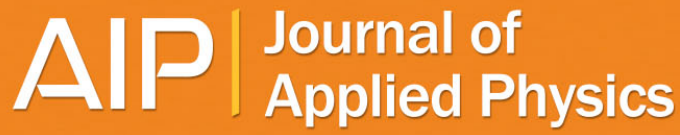

Journal of Applied Physics is pleased to announce André Anders as its new Editor-in-Chief 


\title{
Microscopic calculations of linear and quadratic optical response in model Langmuir-Blodgett multilayers
}

\author{
M. in het Panhuis and R. W. Munn \\ Department of Chemistry, UMIST, Manchester M60 1QD, United Kingdom
}

(Received 30 September 1999; accepted 21 January 2000)

\begin{abstract}
Microscopic calculations are reported of linear and quadratic nonlinear optical response in Langmuir-Blodgett films. The films are modeled as ordered layers of molecules that can tilt from the vertical and have a higher polarizability and first hyperpolarizability in the head than in the tail. The local electric field varies little after the first layer and negligibly after the second, which simplifies the analysis. Tilted two-layer $Y$-type films can have quadratic susceptibility components as large as $X$ - or Z-type ones. Overall the results provide a systematic account of the linear and nonlinear optical properties as a function of molecular and film structure that should assist interpretation of experimental results. (C) 2000 American Institute of Physics.
\end{abstract}

[S0021-9606(00)70115-2]

\section{INTRODUCTION}

Langmuir-Blodgett films are often characterized by linear and nonlinear optical measurements. These serve two main purposes. One is to assess the possible usefulness of the films for applications, usually in nonlinear optics. The other is to deduce information about molecular orientation in the films. In either case, a microscopic theory of the optical response is desirable. It provides an understanding that is helpful to guide preparation of films with improved properties for applications and that is necessary to deduce reliable information on molecular orientation.

The basic ingredients of the required microscopic theory are well known. They are the molecular linear or nonlinear optical response, described by the polarizability and hyperpolarizability, and the local electric field in the film. The molecular response is required relative to an axis system defined relative to the film, typically with $z$-axis normal to the layers. Molecules that form Langmuir-Blodgett films are typically elongated and can often be treated as having roughly axial symmetry. Then conversion of the molecular response tensor components from the molecular axes to the film axes depends only on the tilt $\theta$ of the molecular long axis away from the $z$-axis. Given the local field, the tilt angle can be deduced by comparing the molecular and film responses. However, the local field is seldom treated rigorously, and is often ignored.

A theoretical treatment of the linear optical response of molecular layers was developed by Philpott. ${ }^{1}$ This relied on summing dipolar interactions in a planewise fashion, which shows that interactions between layers fall off exponentially with separation. ${ }^{2,3}$ This work treated molecules as points and was not applied to Langmuir-Blodgett films. Later work calculated planewise dipole sums for molecules treated as sets of points, showing that the exponential fall off set in only four layers with at least one intervening layer. This approach was extended to model Langmuir-Blodgett films and used to calculate linear and nonlinear optical responses as a function of tilt. ${ }^{4,5}$ The treatment ignored interactions between layers, thus effectively treating only a monolayer, and assumed a uniform distribution of response over the molecules. Further work explored the effect of different nonuniform distributions of a fixed total polarizability on the linear optics of Langmuir-Blodgett films. ${ }^{6}$

From this survey it is apparent that there are significant gaps in our detailed understanding even of a highly simplified model Langmuir-Blodgett film. In particular, there are no proper calculations for multilayers, and hence inadequate insight into the differences between $X$-, $Y$-, and $Z$-type films. Nor are there calculations for nonuniform hyperpolarizability, which is likely to be significant for many typical filmforming molecules designed to have a highly active chromophore as the "head" with a relatively inactive elongated "tail." The present article reports calculations that remedy these omissions. We have explored the local fields, refractive indices, and quadratic susceptibilities for model film structures of molecules with model responses as a function of the angle of tilt, the number of layers, and the sequence of layers. Even this rather limited set of parameters generates extensive information that we analyze in order to bring out generic features likely to be useful in interpreting the measured optical response of Langmuir-Blodgett films.

\section{MODEL}

The structure of one layer in a film is defined relative to a reference structure in which the molecules are oriented perpendicular to the layer. Two reference structures have been used in previous work: one with triangular close packing and one with square packing, ${ }^{4-6}$ each of which has experimental support. ${ }^{7,8}$ These are very similar except in quantitative detail, but the square packing is somewhat simpler conceptually and is easier to handle algebraically, especially once the molecules are allowed to tilt. Two directions of tilt were treated previously: one in the plane containing nearestneighbor molecules and one containing next-nearestneighbors. ${ }^{4-6}$ Molecular dynamics simulations indicate a preference for next-nearest-neighbor tilt starting from trian- 
gular packing, ${ }^{9}$ as might be expected since there is more room to tilt in that direction, but systematic studies show that coupling between tilt and lattice distortion plays an important role in determining film structure and phase transitions. $^{10,11}$ In practice, nearest-neighbor and nextnearest-neighbor tilt produce very similar results, but nextnearest-neighbor tilt from triangular packing and nearestneighbor tilt from square parking both produce monoclinic structures that are simpler conceptually and easier to handle algebraically than the triclinic structures produced by the alternative directions of tilt. Hence in the present work we start from triangular packing and next-nearest-neighbor tilt to yield the simplest representative film structures. Within each layer we treat each molecule as a string of $s$ spherical beads or submolecules labeled $j=1, \ldots, s$. This serves to represent the elongated molecules that typically form LangmuirBlodgett films. From previous work, a value $s=5$ is adequate, since larger values yield little change. ${ }^{4}$ Films consisting of molecules with uniform and nonuniform distributed molecular response are treated. To represent the distribution of polarizability and first hyperpolarizability within a molecule, we allow one bead to have a higher response than the others. Typically this bead would be the chromophore contained in a hydrophilic head group, while the other beads constitute a hydrophobic tail. Identifying a head group makes it easier to identify the relative orientation of a layer, although even molecules with uniform response must have a specified axis direction in order to have nonzero first hyperpolarizability and hence nonzero quadratic nonlinear optical (NLO) response. This becomes significant once multilayers are considered. When successive layers are identical, we have a model $X$ - or Z-type film (see Fig. 1), which is necessarily NLO-active, if the molecules are active. When successive layers alternate in the direction perpendicular to the film, we have a model $Y$-type film, which is necessarily NLO-inactive for an even number of layers and zero tilt (normal packing). For $Y$-type bilayers we can consider two different arrangements, with the heads apart [as in Fig. 1(a)] or with the heads together. Then both these $Y$-type arrangements are NLO-inactive regardless of the number of $Y$-type bilayers. For tilted films we consider two types of packing. In the first packing, the molecules in successive layers are tilted in the same direction, so that the molecular axes are parallel or antiparallel (both referred to as parallel packing for brevity). In the other packing the molecules in successive layers are tilted in opposite directions (referred to as herringbone packing). A parallel packing can be arranged in various ways depending on which submolecule we chose to rotate about. For example, rotation about the last submolecule in one layer and the first submolecule in the adjacent layer creates a different arrangement compared with rotation about the first submolecule in each layer. In this work we have chosen the first example resulting in the parallel arrangement shown in Fig. 1(b). For multilayers, as explained later, it is convenient to treat molecules in all layers that interact significantly as belonging to one crystallographic unit cell, which may be larger than the primitive unit cell (see Sec. IV).

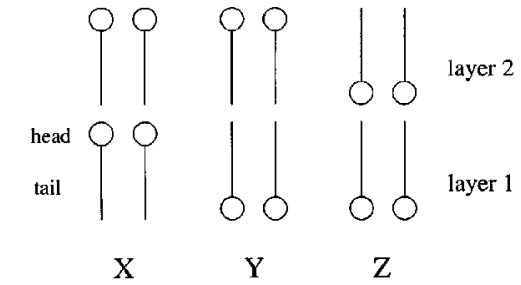

(a)

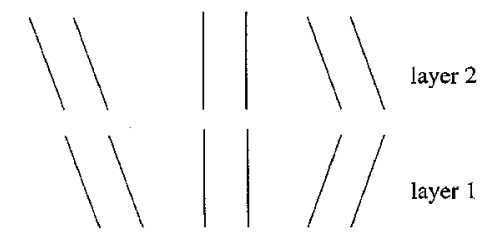

(b)

parallel normal herringbone

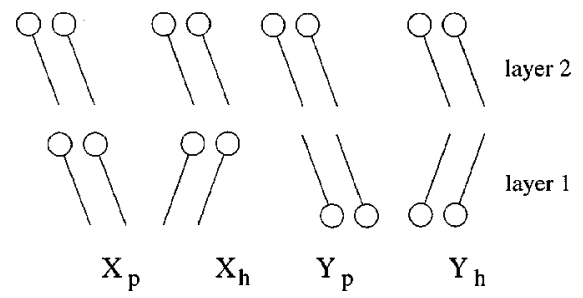

(c)

FIG. 1. Pictorial representation of monolayer arrangement in (a) different types of Langmuir-Blodgett films, (b) molecular packing, and (c) tilted films in different packing. The subscripts " $p$ " and " $h$ " refer to parallel and herringbone packing, respectively.

\section{THEORY}

Unit cell $\lambda$ in layer $g$ contains one molecule, by construction, with submolecules $j$, so that $(\lambda g j)$ defines a unique submolecule at position $\mathbf{r}_{\lambda g j}$. Translational symmetry within a layer means that molecular properties are independent of $\lambda$. The submolecules have polarizabilities $\alpha_{g j}$ and first hyperpolarizabilities $\beta_{g j}$, and the volume per molecule is $v$.

A uniform electric field $\boldsymbol{E}^{0}$ is applied to the film, consistent with applying a light wave of wavelength much greater than the unit cell edge and the film thickness. We first consider linear response. Submolecule $g j$ then acquires a dipole moment given by

$$
\boldsymbol{p}_{g j}=\boldsymbol{\alpha}_{g j} \cdot \boldsymbol{F}_{g j},
$$

where $\boldsymbol{F}_{g j}$ is the local polarizing electric field, which is independent of $\lambda$. For $N$ layers, the linear polarization is given by

$$
\boldsymbol{P}^{(1)}=\sum_{g j} \boldsymbol{p}_{g j} / N v .
$$

This quantity relates the usual macroscopic field $\boldsymbol{E}$ to $\boldsymbol{E}^{0}$ through $^{6}$

$$
\boldsymbol{E}=\boldsymbol{E}^{0}-\boldsymbol{n}\left(\boldsymbol{n} \cdot \boldsymbol{P}^{(1)}\right) / \epsilon_{0},
$$

where $\boldsymbol{n}$ is the unit vector normal to the film. The polarization is also related to the macroscopic field through

$$
\boldsymbol{P}^{(1)}=\boldsymbol{\epsilon}_{0} \boldsymbol{\chi}^{(1)} \cdot \boldsymbol{E},
$$


where $\boldsymbol{\chi}^{(1)}$ is the linear electric susceptibility, one of the targets of the present calculations. It yields the relative permittivity $\boldsymbol{\epsilon}_{r}$ as $\mathbf{1}+\boldsymbol{\chi}^{(1)}$, whence the refractive index $n_{e}$ in a direction defined by unit vector $\boldsymbol{e}$ is obtainable as

$$
1 / n_{e}^{2}=\boldsymbol{e} \cdot\left(\boldsymbol{\epsilon}_{r}\right)^{-1} \cdot \boldsymbol{e} .
$$

The local electric field is given by

$$
\boldsymbol{F}_{g j}=\boldsymbol{E}+\sum_{g^{\prime} j^{\prime}} \boldsymbol{L}_{g j, g^{\prime} j^{\prime}} \cdot \boldsymbol{p}_{g^{\prime} j^{\prime}} / \boldsymbol{\epsilon}_{0} N v .
$$

Here $\boldsymbol{L}_{g j, g^{\prime} j^{\prime}}$ is a planewise Lorentz-factor tensor given by ${ }^{6}$

$$
\boldsymbol{L}_{g j, g^{\prime} j^{\prime}}=\boldsymbol{T}_{g j, g^{\prime} j^{\prime}}+\boldsymbol{n} \boldsymbol{n} \boldsymbol{\delta}_{g g^{\prime}},
$$

where $\boldsymbol{T}_{g j, g^{\prime} j^{\prime}}$ is the dimensionless planewise dipole tensor sum

$$
\boldsymbol{T}_{g j, g^{\prime} j^{\prime}}=\left.\frac{N v}{4 \pi} \sum_{\lambda^{\prime},\left(\lambda g \neq \lambda^{\prime} g^{\prime}\right)} \nabla \nabla\left(\frac{1}{r}\right)\right|_{\mathbf{r}=\mathbf{r}_{\lambda g j^{\prime}}-\mathbf{r}_{\lambda^{\prime} g^{\prime} j^{\prime}}} .
$$

This sum is independent of $\lambda$ because translational symmetry ensures that the summand depends only on the difference $\lambda-\lambda^{\prime}$. The restriction on the sum excludes contributions from coincident points $\left(\lambda g=\lambda^{\prime} g^{\prime}\right.$ with $\left.j=j^{\prime}\right)$ and from different submolecules on the same molecule $\left(\lambda g=\lambda^{\prime} g^{\prime}\right.$ with $\left.j \neq j^{\prime}\right)$, since a molecule does not polarize itself.

Substitution in Eq. (6) for the local fields from Eq. (1) gives a set of equations that can be solved to relate the local fields to the macroscopic field. The result can be expressed as follows:

$$
\boldsymbol{F}_{g j}=\boldsymbol{d}_{g j} \cdot \boldsymbol{E},
$$

where $\boldsymbol{d}_{g j}$ is the local-field tensor given by

$$
\begin{aligned}
& \boldsymbol{d}_{g j}=\sum_{g^{\prime} j^{\prime}} \boldsymbol{D}_{g j, g^{\prime} j^{\prime}}, \\
& \boldsymbol{D}_{g j, g^{\prime} j^{\prime}}=(\boldsymbol{I}-\boldsymbol{L} \cdot \boldsymbol{A})_{g j, g^{\prime} j^{\prime}}^{-1} .
\end{aligned}
$$

Here the matrices on the right hand side are of order $3 \mathrm{Ns}$, where $N$ is the number of unit cells per crystallographic unit cell and $s$ the number of submolecules per molecule. $\boldsymbol{I}$ is the unit matrix with $3 \times 3$ submatrices $\mathbf{l} \delta_{g g^{\prime}} \delta_{j j^{\prime}} ; \boldsymbol{L}$ is the Lorentz-factor matrix with $3 \times 3$ submatrices $\boldsymbol{L}\left(g j, g^{\prime} j^{\prime}\right)$; and $\boldsymbol{A}$ is the polarizability matrix with $3 \times 3$ submatrices $\boldsymbol{a}(g j) \delta_{g g^{\prime}} \delta_{j j^{\prime}}$, where $\boldsymbol{a}(g j)$ is the dimensionless reduced polarizability $\boldsymbol{\alpha}(g j) / \epsilon_{0} N v$. From Eqs. (2), (4), and (9), the linear susceptibility can be expressed as

$$
\boldsymbol{\chi}^{(1)}=\sum_{g j} \boldsymbol{a}_{g j} \cdot \boldsymbol{d}_{g j},
$$

which with Eqs. (10) and (11) leads to

$$
\boldsymbol{\chi}^{(1)}=\sum_{g j, g^{\prime} j^{\prime}}\left(\boldsymbol{A}^{-1}-\boldsymbol{L}\right)_{g j, g^{\prime} j^{\prime}}^{-1} .
$$

These results are developments of those derived previously, ${ }^{6,12}$ with now the macroscopic electric field defined for the whole film rather than for each individual layer (or for a film that comprised a single layer). As a consequence, the results are algebraically the same as for bulk dielectric response in molecular crystals, with $g$ replacing the usual index $k$ for different molecules in the unit cell (which is absent because our model has only one molecule in the primitive unit cell). In practice, the interactions between layers fall off so rapidly that coupling may be insignificant beyond two or three layers, ${ }^{4}$ as the calculations reported below confirm. In that case it suffices to restrict the sum over layers to those that interact significantly, with $N$ suitably altered. Formally, this occurs because the matrices for the complete film in Eq. (13) reduce to a bordered form such that $g^{\prime}$ is restricted to a few values either side of $g$; for a given value of $g-g^{\prime}$ (i.e., along the diagonal or one of its borders), the values are the same, and so the sum over $g$ gives the same values for all $g$ except when $g$ lies close to the film surface. A further consequence is that it is possible to evaluate the results for $N$ layers by treating the multilayer film as a single layer consisting of unit cells $N$ layers thick that contain $N$ molecules (see below).

We now consider quadratic response. This leads to a quadratic polarization $\boldsymbol{P}^{(2)}$ that is related to the macroscopic electric field by

$$
\boldsymbol{P}^{(2)}=\boldsymbol{\epsilon}_{0} \boldsymbol{\chi}^{(2)}: \boldsymbol{E} \boldsymbol{E},
$$

where $\boldsymbol{\chi}^{(2)}$ is the quadratic electric susceptibility. The foregoing development of the previous treatments of linear response is readily extended to the previous treatment of nonlinear response, ${ }^{5}$ to yield

$$
\boldsymbol{\chi}^{(2)}=\sum_{g j} \boldsymbol{b}_{g j} \vdots \boldsymbol{d}_{g j} \boldsymbol{d}_{g j} \boldsymbol{d}_{g j},
$$

where $\boldsymbol{b}_{g j}=\beta_{g j} / \epsilon_{0} N v$ is a reduced first hyperpolarizability. This expression uses the linear local-field tensors but accounts fully for the nonlinear local field, as indicated by the presence of three local field tensors when two might have been expected for quadratic response: the additional factor comes from combining quadratic response to the linear local field with linear response to the nonlinear local field.

\section{CALCULATION DETAILS}

An existing program developed locally was used for our calculations. In treating multilayers, it is convenient to use a multilayer unit cell structure that is the same thickness as the whole multilayer. This automatically handles all the interactions between layers.

The multilayer unit cell for zero tilt consists of $N$ layer unit cells arranged perpendicular to the layers. The unit cell then contains one molecule. Thus, the submolecule fractional coordinates of a multilayer unit cell with $N=1$ are $[0,0,(j$ $-1) / s]$, where $j=1, \ldots, s$. For a multilayer unit cell with $N$ $>1$ the submolecular fractional coordinates are

$$
\begin{aligned}
& \text { first layer: } 0,0,(j-1) / N s, \\
& \text { subsequent layers: } 0,0,[s(G-1)+(j-1)] / N s,
\end{aligned}
$$

where $j=1, \ldots, s$ and $G=2, \ldots, N$ labels the layers. Multilayer effects were studied by varying the number of layers between one and five, with a molecule consisting of two submolecules and no tilt. The molecular response was anisotropic with the response along the molecular axis (in the $z$ direction) linearly proportional to the number of submol- 
TABLE I. Components of the reduced polarizability $a_{\alpha \beta}$ and first hyperpolarizability $b_{\alpha \beta \gamma} / \mathrm{pm} \mathrm{V}^{-1}$ as a function of tilt $\theta$ from the vertical. Only independent nonzero components are shown.

\begin{tabular}{|c|c|c|c|c|c|c|c|c|c|c|}
\hline \multirow[b]{2}{*}{$\theta$} & \multicolumn{4}{|c|}{$a_{\alpha \beta}$} & \multicolumn{6}{|c|}{$b_{\alpha \beta \gamma}$} \\
\hline & $x x$ & $x z$ & $y y$ & $z z$ & $x x x$ & $x y y$ & $x z z$ & $z x x$ & $z y y$ & $z z z$ \\
\hline $0^{\circ}$ & 0.200 & 0 & 0.200 & 1.000 & 0 & 0 & 0 & 3.8 & 3.8 & -19.0 \\
\hline $20^{\circ}$ & 0.294 & 0.257 & 0.200 & 0.906 & -2.68 & -1.30 & 7.88 & 0.23 & 3.57 & -14.51 \\
\hline $40^{\circ}$ & 0.531 & 0.394 & 0.200 & 0.669 & 0.75 & -2.44 & 9.02 & -6.71 & 2.91 & -4.93 \\
\hline
\end{tabular}

ecules, as described in previous work. ${ }^{4,5}$ The values for the reduced polarizability and first hyperpolarizability per molecule are given in Table I. These quantities were kept constant to allow comparison between results with varying $N$.

Once tilt is admitted, it is more convenient to use a monoclinic multilayer unit cell that consists of two unit cells arranged perpendicular to the layers (only the first two layers interact significantly as we will show in Sec. V A). The layer unit cell contains two molecules each consisting of five submolecules. The submolecule coordinates of the molecules are

layer 1: $0,0,(j-1) / 10$ and $0.5,0.5,(j-1) / 10$,

layer 2: $0,0,[5+(j-1)] / 10$ and $0.5,0.5$,

$$
[5+(j-1)] / 10,
$$

where $j=1, \ldots, 5$. The molecules were tilted in the $x z$ plane at two angles, $20^{\circ}$ and $40^{\circ} . X-, Y$-, and Z-type films with uniformly and nonuniformly distributed molecular response were used. In films with a nonuniform distribution, the molecular response of the head submolecule was taken as four times the total response of the submolecules forming the tail, in all directions. Note, this is an extreme case of distributing the response, which differs with previous ${ }^{13}$ and future work. ${ }^{14}$ The total reduced polarizability and first hyperpolarizability in the multilayer unit cell as a function of angle of tilt are given in Table I. Since tilt occurs in the $x z$ plane, the yy component of the reduced polarizability and first hyperpolarizability are constant. For $Y$-type films it is necessary to transform the molecular response for one layer in the multilayer unit cell. For example, for a parallel packed $Y$-type film, $Y_{p}$, the directions of the $x$ - and $z$-axes have to be changed in one layer, whereas for a herringbone packed $Y$-type film, $Y_{h}$, only the direction of the $z$-axis has to be altered. Axis directions were changed using appropriate transformation matrices. It is important to realize that, regardless of axis transformations, the film Cartesian axes are defined independent of the type of film and its packing.

\section{RESULTS}

The different types of monolayer arrangement for $X$-, $Y$ and Z-type films were described in Sec. III. In the following sections the results for the local-field tensor, refractive index, and quadratic susceptibility of untilted films are compared to two angles of tilt, $20^{\circ}$ and $40^{\circ}$. Films with uniform and nonuniform molecular response are compared. The total reduced polarizability and first hyperpolarizability do not change with the angle of tilt. The effect of packing the layers in the unit cell on the results is also explored. For tilted films, the parallel and herringbone packing reduce to the normal packing for zero tilt. The arrangement or sequence of the layers in the multilayer unit cell has an effect on the linear and nonlinear optical properties, since the interacting submolecules of the head and tail are located at a different distance. For example, the distance between heads is larger in a $Y_{p}$ film compared with a $Y_{h}$ film.

\section{A. Number of layers}

Figure 2 shows the variation of the $z z$ component of the local field, the refractive index in the $z$ direction, and the $z z z$ component of the quadratic susceptibility with the number of
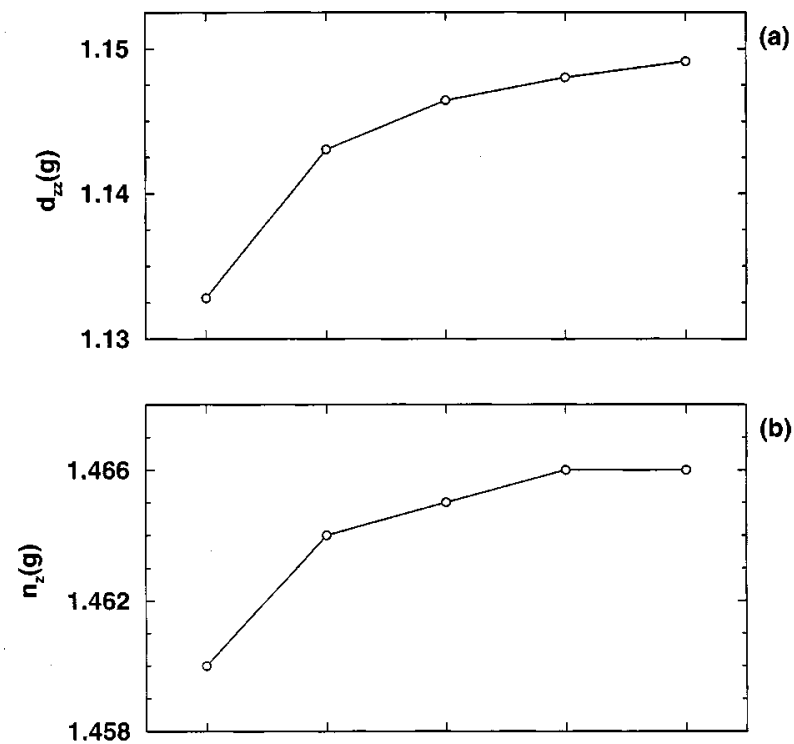

(b)

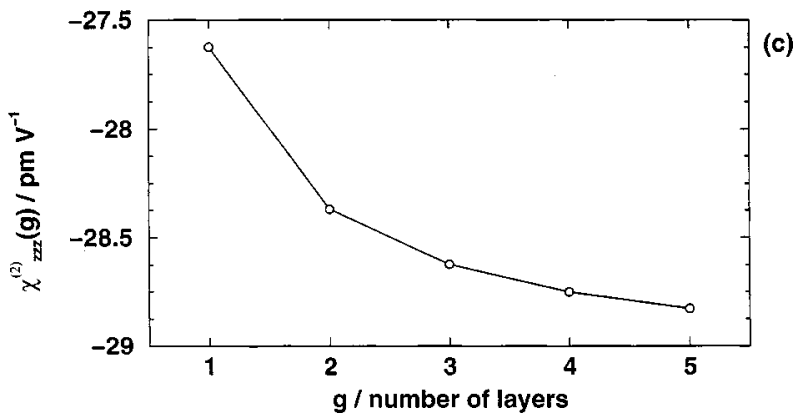

FIG. 2. Dependence of (a) local field tensor ( $z z$ component), (b) refractive index ( $z$ component), and (c) quadratic susceptibility ( $z z z$ component) on the number of monolayers (not tilted) in the multilayer unit cell for Langmuir-Blodgett films with uniform distributed polarizability and first hyperpolarizability. 
TABLE II. Components of the local field tensor $d_{\alpha \beta}$ as a function of tilt $\theta$ from the vertical in Langmuir-Blodgett films. Comparison between independent nonzero components (in the film Cartesian axes) of films with uniform molecular response. Subscripts indicate the packing in the multilayer unit cell, $p$ refers to parallel and $h$ refers to herringbone packing.

\begin{tabular}{crrllll}
\hline \hline & & \multicolumn{5}{c}{$\alpha \beta$} \\
\cline { 3 - 7 } Type & $\theta$ & $x x$ & $x z$ & $y y$ & $z x$ & $z z$ \\
\hline$X_{p}$ & $0^{\circ}$ & 1.109 & 0 & 1.109 & 0 & 1.024 \\
& $20^{\circ}$ & 1.197 & 0.265 & 1.103 & 0.071 & 1.144 \\
& $40^{\circ}$ & 1.354 & 0.383 & 1.090 & 0.373 & 1.471 \\
$X_{h}$ & $0^{\circ}$ & 1.109 & 0 & 1.109 & 0 & 1.024 \\
& $20^{\circ}$ & 1.150 & 0 & 1.103 & 0 & 1.149 \\
& $40^{\circ}$ & 1.213 & 0 & 1.090 & 0 & 1.475 \\
$Y_{p}$ & $0^{\circ}$ & 1.109 & 0 & 1.109 & 0 & 1.024 \\
& $20^{\circ}$ & 1.197 & 0.265 & 1.103 & 0.071 & 1.144 \\
& $40^{\circ}$ & 1.354 & 0.383 & 1.090 & 0.373 & 1.471 \\
$Y_{h}$ & $0^{\circ}$ & 1.109 & 0 & 1.109 & 0 & 1.024 \\
& $20^{\circ}$ & 1.150 & 0 & 1.103 & 0 & 1.144 \\
& $40^{\circ}$ & 1.213 & 0 & 1.090 & 0 & 1.475 \\
\hline \hline
\end{tabular}

layers in the multilayer unit cell. The other components of these properties show similar dependence. Increasing the number of layers per multilayer unit cell from one to two shows the largest change in the properties. Multilayer unit cells with three or more layers result in a smaller change. This is in agreement with previous work ${ }^{4}$ using a single layered multilayer unit cell which established that significant dipolar interactions extend only to adjacent layers.

\section{B. Uniform molecular response}

\section{Local-field tensor}

The local field tensor components for LangmuirBlodgett films with uniform molecular response are shown in Table II. Since tilt occurs in the $x z$ plane, $d_{y y}$ remains approximately constant for all angles of tilt and all types of films. Both $d_{x x}$ and $d_{z z}$ increase with increasing angle of tilt: they follow the tilt. The component $d_{x x}$ is independent of the type of film but dependent on the type of molecular packing, changing more for parallel packing than for herringbone packing. $d_{z z}$ is independent of the type of film and packing. Introducing tilt leads to off-diagonal components in parallel packed films. This is not observed for herringbone packed films. Each individual layer in these films has off-diagonal components, but they are equal and opposite for alternating layers. Thus, the sum amounts to zero, as expected for the overall orthogonal unit cell of the herringbone packing.

\section{Refractive index}

The refractive indices were calculated using Eq. (5), with the relative permittivity tensor constructed from the linear susceptibility tensor. The results that we discuss are given in the film Cartesian axes rather than the principal optical axes. We chose to tabulate the refractive indices in these axes since they are the linear quantities most likely to be measured.

As expected, $n_{y}$ is independent of tilt (see Table III),
TABLE III. Refractive index $n$ of Langmuir-Blodgett films in different directions as a function of tilt $\theta$ from the vertical. Comparison between components along the film Cartesian axes of films with uniform molecular response. Subscripts indicate the packing in the multilayer unit cell, $p$ refers to parallel and $h$ refers to herringbone packing.

\begin{tabular}{|c|c|c|c|c|}
\hline \multirow[b]{2}{*}{ Type } & \multirow[b]{2}{*}{$\theta$} & \multicolumn{3}{|c|}{ Direction } \\
\hline & & $x$ & $y$ & $z$ \\
\hline \multirow[t]{3}{*}{$X_{p}$} & $0^{\circ}$ & 1.105 & 1.105 & 1.423 \\
\hline & $20^{\circ}$ & 1.142 & 1.105 & 1.415 \\
\hline & $40^{\circ}$ & 1.257 & 1.104 & 1.344 \\
\hline \multirow[t]{3}{*}{$X_{h}$} & $0^{\circ}$ & 1.105 & 1.105 & 1.423 \\
\hline & $20^{\circ}$ & 1.142 & 1.105 & 1.451 \\
\hline & $40^{\circ}$ & 1.255 & 1.104 & 1.464 \\
\hline \multirow[t]{3}{*}{$Y_{p}$} & $0^{\circ}$ & 1.105 & 1.105 & 1.423 \\
\hline & $20^{\circ}$ & 1.142 & 1.105 & 1.415 \\
\hline & $40^{\circ}$ & 1.257 & 1.104 & 1.344 \\
\hline \multirow[t]{3}{*}{$Y_{h}$} & $0^{\circ}$ & 1.105 & 1.105 & 1.423 \\
\hline & $20^{\circ}$ & 1.142 & 1.105 & 1.451 \\
\hline & $40^{\circ}$ & 1.255 & 1.104 & 1.464 \\
\hline
\end{tabular}

since tilt occurs in the $x z$ plane. On the other hand, $n_{x}$ increases with the angle of tilt and is independent of the type of film, while $n_{z}$ decreases with tilt for parallel packed films, but increases with tilt for herringbone packed films. $X$ - and $Y$-type films with the same packing behave similarly since they are equal in composition except for the specified axis direction. For example, in a $X_{h}$-type film the $x$-axis directions of the layers are opposite, while in a $Y_{h}$-type film, the $z$-axis directions of the layers are opposite. Thus the molecular response of one of the layers is transformed in the $x$ direction for a $X_{p}$-type and in the $z$ direction for a $Y_{h}$-type film. This leads to a sign change in the off-diagonal components of the molecular polarizability for one of the layers. Since tilt occurs in the $x z$ plane a sign change in either $x$ or $z$ leads to similar results in the linear susceptibility. Parallel packed films have nonzero off-diagonal components for the local field tensor. Furthermore, $d_{z z}$ and the reduced polarizability are independent of the type of film. Hence, the decrease in $n_{z}$ for parallel packed films and the increase in $n_{z}$ for herringbone packed films can be explained through the effect of the off-diagonal local-field tensor components. Nonzero off-diagonal local-field tensor components increase with the tilt angle, leading to a decrease in $n_{z}$ with tilt, whereas zero off-diagonal components lead to an increase in $n_{z}$ with tilt.

\section{Quadratic susceptibility}

The results for the quadratic susceptibility are given in Table IV. First we consider untilted films. $X$ - and $Y$-type films are not equivalent, and this is reflected in the results. An untilted $Y$-type film is centrosymmetric, with a center of inversion, and is necessarily NLO-inactive. Tilted films have different optical response. For $X$-type film with $20^{\circ}$ tilt we note that all nonzero components are larger than the reduced first hyperpolarizability except for the $z z z$ component. This is also valid for $40^{\circ}$ tilt except that here we observe a sign change for $x x x$ and $z z z$. This behavior can also be seen for 
TABLE IV. Components of the quadratic susceptibility $\chi_{\alpha \beta \gamma} / \mathrm{pm} \mathrm{V}^{-1}$ of Langmuir-Blodgett films as a function of tilt $\theta$ from the vertical. Comparison between independent nonzero components (in the film Cartesian axes) of films with uniform molecular response. Subscripts indicate the packing in the multilayer unit cell, $p$ refers to parallel and $h$ refers to herringbone packing.

\begin{tabular}{cccccccc}
\hline \hline & & \multicolumn{7}{c}{$\alpha \beta \gamma$} \\
\cline { 3 - 8 } Type & $\theta$ & $x x x$ & $x y y$ & $x z z$ & $z x x$ & $z y y$ & $z z z$ \\
\hline$X_{p}$ & $0^{\circ}$ & 0 & 0 & 0 & 4.78 & 4.78 & -20.52 \\
& $20^{\circ}$ & -4.39 & -1.58 & 11.24 & 0.82 & 4.54 & -13.66 \\
& $40^{\circ}$ & -7.05 & -2.64 & 15.72 & -7.30 & 3.97 & 2.22 \\
$X_{h}$ & $0^{\circ}$ & 0 & 0 & 0 & 4.78 & 4.78 & -20.52 \\
& $20^{\circ}$ & 0 & 0 & 0 & -3.61 & 4.54 & -13.60 \\
& $40^{\circ}$ & 0 & 0 & 0 & -18.68 & 3.94 & 2.80 \\
$Y_{p}$ & $0^{\circ}$ & 0 & 0 & 0 & 0 & 0 & 0 \\
& $20^{\circ}$ & 0 & 0 & 0 & 0 & 0 & 0 \\
& $40^{\circ}$ & 0 & 0 & 0 & 0 & 0 & 0 \\
$Y_{h}$ & $0^{\circ}$ & 0 & 0 & 0 & 0 & 0 & 0 \\
& $20^{\circ}$ & -3.69 & -2.39 & 13.67 & 0 & 0 & 0 \\
& $40^{\circ}$ & 7.46 & -4.12 & 14.62 & 0 & 0 & 0 \\
\hline \hline
\end{tabular}

$Y$-type films, except that some components cancel, owing to opposing contributions from the individual layers. In a tilted herringbone packed $X$-type film, $X_{h}$, the $x$-axis direction is transformed for one of the layers, resulting in components with an odd number of $x$ becoming zero. As mentioned before, this occurs because individual layer contributions cancel. Tilting a $Y$-type film into a parallel packing changes the symmetry to a mirror plane. Since this plane contains the $x$ and $y$-axes, individual layer components with an odd number of $z$ indices are equal and opposite. Thus, the film total (the sum over the layer components) has to be zero. Tilting a $Y$-type film into a herringbone packing does not alter the symmetry, and the film remains centrosymmetric, as the results show.

\section{Effect of molecular packing}

We already mentioned that parallel and herringbone packed films reduce to the normal packing for zero angle tilt. This can be used to assess the effect of molecular packing. The $x x$ components of the local field tensor are larger in the parallel packing than in the herringbone packing. Nonzero off-diagonal components are only observed in parallel packed films. All other components are equal for both types of packing. The $z$ component of the refractive index increases with tilt for herringbone packed films and decreases with tilt for parallel packed films. This is a result of nonzero off-diagonal local-field tensor components for parallel packed films. Figure 3 shows a comparison between the $z z z$, $z x x$, and $x z z$ components of $\chi^{(2)}$ as a function of angle of tilt for $X$-type films. The figure shows that the $z z z$ component follows the same pattern for both packings. As the angle of tilt is increased, $\chi^{(2)}$ become less negative and for a tilt of $40^{\circ}$ a sign change is observed. The $z x x$ component is more affected by tilt for herringbone packing than for parallel
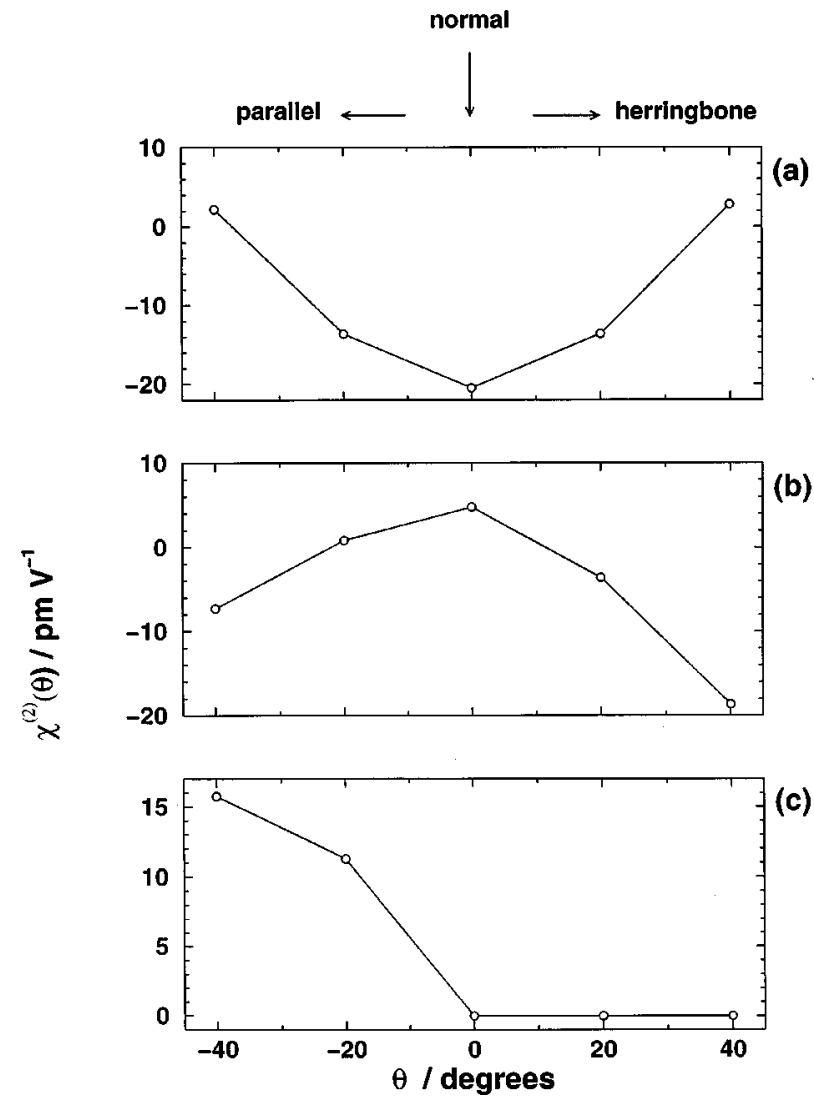

FIG. 3. Variation of quadratic susceptibility with tilt in the $x z$ plane for Langmuir-Blodgett films with uniform distributed molecular response. (a) $z z z$, (b) $z x x$, and (c) $x z z$ components. Normal, parallel, and herringbone refer to different ways of packing in the multilayer unit cell as explained in the text.

packing. A herringbone packed film has a zero $x z z$ component, while in a parallel packed film a steady increase with tilt is noted.

The difference in the magnitude of the $\chi^{(2)}$ components can be attributed to nonzero off-diagonal components in the local-field tensor for parallel packed films. Off-diagonal components are absent for herringbone packed films. Detailed analysis reveals that the impact of off-diagonal components is smaller for the $z z z$ component than for the $z x x$ component, which is in excellent agreement with Fig. 3.

\section{Nonuniform molecular response}

We now consider Langmuir-Blodgett film with a nonuniform distributed molecular response. We start with a comparison between $X$-, $Y$-, and $Z$-type packings. According to our convention, a $Y$-type film consists of two monolayers arranged in such a manner that the polar heads are furthest apart (see Fig. 1). As was mentioned in Sec. II a different arrangement exists in which the polar heads are closest together, referred to as $Y^{\prime}$-type. We compare these two arrangements.

\section{Local-field tensor}

The results for the local-field tensor of parallel and herringbone packed films are shown in Table V. For all types of films $d_{x x}$ and $d_{z z}$ increase with tilt, while $d_{y y}$ decreases 
TABLE V. Components of the local field tensor $d_{\alpha \beta}$ as a function of tilt $\theta$ from the vertical in parallel and herringbone packed Langmuir-Blodgett films. Comparison between independent nonzero components (in the film Cartesian axes) of films with nonuniform molecular response. $Y^{\prime}$ indicates $Y$-type films with the heads in successive layers at the shortest distance, whereas $Y$ indicates $Y$-type films with the heads at the largest distance.

\begin{tabular}{|c|c|c|c|c|c|c|c|}
\hline \multirow[b]{2}{*}{ Packing } & \multirow[b]{2}{*}{ Type } & \multirow[b]{2}{*}{$\theta$} & \multicolumn{5}{|c|}{$\alpha \beta$} \\
\hline & & & $x x$ & $x z$ & $y y$ & $z x$ & $z z$ \\
\hline \multirow[t]{12}{*}{ Parallel } & $X$ & $0^{\circ}$ & 1.125 & 0 & 1.125 & 0 & 1.031 \\
\hline & & $20^{\circ}$ & 1.176 & 0.132 & 1.115 & 0.055 & 1.089 \\
\hline & & $40^{\circ}$ & 1.288 & 0.211 & 1.093 & 0.271 & 1.273 \\
\hline & $Y$ & $0^{\circ}$ & 1.126 & 0 & 1.126 & 0 & 1.029 \\
\hline & & $20^{\circ}$ & 1.177 & 0.133 & 1.116 & 0.054 & 1.087 \\
\hline & & $40^{\circ}$ & 1.299 & 0.215 & 1.096 & 0.260 & 1.259 \\
\hline & $Y^{\prime}$ & $0^{\circ}$ & 1.123 & 0 & 1.123 & 0 & 1.033 \\
\hline & & $20^{\circ}$ & 1.173 & 0.133 & 1.113 & 0.057 & 1.095 \\
\hline & & $40^{\circ}$ & 1.269 & 0.209 & 1.087 & 0.281 & 1.298 \\
\hline & Z & $0^{\circ}$ & 1.125 & 0 & 1.125 & 0 & 1.031 \\
\hline & & $20^{\circ}$ & 1.176 & 0.132 & 1.115 & 0.055 & 1.089 \\
\hline & & $40^{\circ}$ & 1.288 & 0.211 & 1.093 & 0.271 & 1.273 \\
\hline \multirow[t]{12}{*}{ Herringbone } & $X$ & $0^{\circ}$ & 1.125 & 0 & 1.125 & 0 & 1.031 \\
\hline & & $20^{\circ}$ & 1.158 & 0.001 & 1.115 & -0.001 & 1.090 \\
\hline & & $40^{\circ}$ & 1.221 & 0.005 & 1.093 & -0.008 & 1.275 \\
\hline & Y & $0^{\circ}$ & 1.126 & 0 & 1.126 & 0 & 1.029 \\
\hline & & $20^{\circ}$ & 1.159 & 0 & 1.116 & 0 & 1.087 \\
\hline & & $40^{\circ}$ & 1.231 & 0 & 1.096 & 0 & 1.259 \\
\hline & $Y^{\prime}$ & $0^{\circ}$ & 1.123 & 0 & 1.123 & 0 & 1.033 \\
\hline & & $20^{\circ}$ & 1.154 & 0 & 1.113 & 0 & 1.095 \\
\hline & & $40^{\circ}$ & 1.193 & 0 & 1.087 & 0 & 1.332 \\
\hline & $Z$ & $0^{\circ}$ & 1.125 & 0 & 1.125 & 0 & 1.031 \\
\hline & & $20^{\circ}$ & 1.158 & -0.001 & 1.115 & 0.001 & 1.090 \\
\hline & & $40^{\circ}$ & 1.221 & -0.005 & 1.093 & 0.008 & 1.275 \\
\hline
\end{tabular}

slightly. $X$ - and Z-type films behave similarly, since they are equivalent in composition except for the direction of their $z$-axis. The distance between head and tail groups in successive monolayers is equal. Thus, similar local-field tensor components for untilted and tilted films are observed for both types of packing. In $Y$-type films the distance between the head and tail groups in successive monolayers is different compared to $X$ - and $Z$-type films. In addition to this, the $z$-axis directions of successive monolayers are opposite. This is reflected in the results. In comparison to $X$ - and $Z$-type films, a $Y$-type film has larger $x x$, similar $x z, y y$ and smaller $z x$ and $z z$ components. This is most prominent for the largest angle of tilt. In contrast to these observations a $Y^{\prime}$-type film shows exactly the opposite; smaller $x x$, similar $x z, y y$ and larger $z x$ and $z z$ components. From these results we deduce the following: $d_{z z}$ varies inversely with the interlayer distance between the heads in $z$ direction, whereas $d_{x x}$ varies directly with the interlayer separation between the heads in the $x$ direction. The observed trends are valid for both types of molecular packing, but the magnitude of the individual components changes with the type of packing. In a herringbone packed $Y$-type film, tilt does not lead to off-diagonal components, owing to the mirror plane. The off-diagonal components of the layers are equal but opposite in sign and
TABLE VI. Refractive index $n$ of parallel and herringbone packed Langmuir-Blodgett films in different directions as a function of tilt $\theta$ from the vertical. Comparison between components along the film Cartesian axes of films with nonuniform molecular response. $Y^{\prime}$ indicates $Y$-type films with the heads in successive layers at the shortest distance, whereas $Y$ indicates $Y$-type films with the heads at the largest distance.

\begin{tabular}{|c|c|c|c|c|c|}
\hline \multirow[b]{2}{*}{ Packing } & \multirow[b]{2}{*}{ Type } & \multirow[b]{2}{*}{$\theta$} & \multicolumn{3}{|c|}{ Direction } \\
\hline & & & $x$ & $y$ & $z$ \\
\hline \multirow[t]{12}{*}{ Parallel } & $X$ & $0^{\circ}$ & 1.128 & 1.128 & 1.239 \\
\hline & & $20^{\circ}$ & 1.165 & 1.125 & 1.236 \\
\hline & & $40^{\circ}$ & 1.300 & 1.118 & 1.216 \\
\hline & $Y$ & $0^{\circ}$ & 1.128 & 1.128 & 1.239 \\
\hline & & $20^{\circ}$ & 1.165 & 1.125 & 1.235 \\
\hline & & $40^{\circ}$ & 1.301 & 1.119 & 1.215 \\
\hline & $Y^{\prime}$ & $0^{\circ}$ & 1.127 & 1.127 & 1.241 \\
\hline & & $20^{\circ}$ & 1.163 & 1.124 & 1.239 \\
\hline & & $40^{\circ}$ & 1.287 & 1.115 & 1.226 \\
\hline & Z & $0^{\circ}$ & 1.128 & 1.128 & 1.239 \\
\hline & & $20^{\circ}$ & 1.165 & 1.125 & 1.236 \\
\hline & & $40^{\circ}$ & 1.300 & 1.118 & 1.216 \\
\hline \multirow[t]{12}{*}{ Herringbone } & $X$ & $0^{\circ}$ & 1.128 & 1.128 & 1.239 \\
\hline & & $20^{\circ}$ & 1.165 & 1.125 & 1.249 \\
\hline & & $40^{\circ}$ & 1.300 & 1.118 & 1.274 \\
\hline & Y & $0^{\circ}$ & 1.128 & 1.128 & 1.239 \\
\hline & & $20^{\circ}$ & 1.165 & 1.125 & 1.248 \\
\hline & & $40^{\circ}$ & 1.301 & 1.119 & 1.270 \\
\hline & $Y^{\prime}$ & $0^{\circ}$ & 1.127 & 1.127 & 1.241 \\
\hline & & $20^{\circ}$ & 1.163 & 1.124 & 1.254 \\
\hline & & $40^{\circ}$ & 1.276 & 1.115 & 1.308 \\
\hline & $Z$ & $0^{\circ}$ & 1.128 & 1.128 & 1.239 \\
\hline & & $20^{\circ}$ & 1.165 & 1.125 & 1.249 \\
\hline & & $40^{\circ}$ & 1.300 & 1.118 & 1.274 \\
\hline
\end{tabular}

thus cancel. Parallel packed $Y$ - and $Y^{\prime}$-type films are centrosymmetric, and hence zero off-diagonal components are expected. The nonzero off-diagonal components are a result of transforming the polarizability tensor in the $x$ and $z$ direction for one of the layers, but this does not lead to any sign changes, as discussed in the previous section. We note that the magnitude of the $x x$ components is larger for parallel packed $X$-, $Y$-, and $Z$-type films than for herringbone packed films. All other components are approximately equal in magnitude. For $Y^{\prime}$-type films the $x x$ component is larger in a parallel packing, but the $z z$ component is largest in the herringbone packing. This becomes more evident for the largest angle of tilt. These differences can be explained in the following way. In $X$ - and $Z$-type films, the distance between the heads is independent of the type of packing. Hence, the change in $d_{x x}$ is due to head-tail interactions. The head-tail distance between adjacent layers is shorter in a herringbone packing resulting in a smaller $d_{x x}$. In a $Y$-type film the head-head distance changes, but since head-head interactions are likely to be small (due to their large separation), these changes are also caused by head-tail interactions. The decrease in $d_{x x}$ for $Y^{\prime}$-type film can be explained along similar lines, since the head-tail distance changes. $d_{z z}$ shows a small increase for $X$ - and $Z$-type films in the herringbone 
TABLE VII. Components of the quadratic susceptibility $\chi_{\alpha \beta \gamma} / \mathrm{pm} \mathrm{V}^{-1}$ of parallel and herringbone packed Langmuir-Blodgett films as a function of tilt $\theta$ from the vertical. Comparison between independent nonzero components (in the film Cartesian axes) of films with nonuniform molecular response. $Y^{\prime}$ indicates $Y$-type films with the heads in successive layers at the shortest distance, whereas $Y$ indicates $Y$-type films with the heads at the largest distance.

\begin{tabular}{|c|c|c|c|c|c|c|c|c|}
\hline \multirow[b]{2}{*}{ Packing } & \multirow[b]{2}{*}{ Type } & \multirow[b]{2}{*}{$\theta$} & \multicolumn{6}{|c|}{$\alpha \beta \gamma$} \\
\hline & & & $x x x$ & $x y y$ & $x z z$ & $z x x$ & $z y y$ & $z z z$ \\
\hline \multirow[t]{12}{*}{ Parallel } & $X$ & $0^{\circ}$ & 0 & 0 & 0 & 3.33 & 3.33 & -7.31 \\
\hline & & $20^{\circ}$ & -7.00 & -4.93 & 3.41 & -2.94 & 2.49 & -5.16 \\
\hline & & $40^{\circ}$ & 51.78 & -9.17 & 0.28 & -7.49 & 0.66 & -2.14 \\
\hline & $Y$ & $0^{\circ}$ & 0 & 0 & 0 & 0 & 0 & 0 \\
\hline & & $20^{\circ}$ & 0 & 0 & 0 & 0 & 0 & 0 \\
\hline & & $40^{\circ}$ & 0 & 0 & 0 & 0 & 0 & 0 \\
\hline & $Y^{\prime}$ & $0^{\circ}$ & 0 & 0 & 0 & 0 & 0 & 0 \\
\hline & & $20^{\circ}$ & 0 & 0 & 0 & 0 & 0 & 0 \\
\hline & & $40^{\circ}$ & 0 & 0 & 0 & 0 & 0 & 0 \\
\hline & $Z$ & $0^{\circ}$ & 0 & 0 & 0 & -3.33 & -3.33 & 7.31 \\
\hline & & $20^{\circ}$ & 7.00 & 4.93 & -3.41 & 2.94 & -2.49 & 5.16 \\
\hline & & $40^{\circ}$ & -51.78 & 9.17 & -0.28 & 7.49 & -0.66 & 2.14 \\
\hline \multirow[t]{12}{*}{ Herringbone } & $X$ & $0^{\circ}$ & 0 & 0 & 0 & 3.33 & 3.33 & -7.31 \\
\hline & & $20^{\circ}$ & -0.005 & -0.004 & 0.016 & -3.98 & 2.48 & -5.14 \\
\hline & & $40^{\circ}$ & 0.35 & -0.025 & 0.066 & -7.64 & 0.60 & -1.98 \\
\hline & $Y$ & $0^{\circ}$ & 0 & 0 & 0 & 0 & 0 & 0 \\
\hline & & $20^{\circ}$ & -5.61 & -5.28 & 4.09 & 0 & 0 & 0 \\
\hline & & $40^{\circ}$ & 59.46 & -9.43 & 9.05 & 0 & 0 & 0 \\
\hline & $Y^{\prime}$ & $0^{\circ}$ & 0 & 0 & 0 & 0 & 0 & 0 \\
\hline & & $20^{\circ}$ & -5.30 & -5.08 & 4.13 & 0 & 0 & 0 \\
\hline & & $40^{\circ}$ & 46.26 & -8.14 & 0.13 & 0 & 0 & 0 \\
\hline & $Z$ & $0^{\circ}$ & 0 & 0 & 0 & -3.33 & -3.33 & 7.31 \\
\hline & & $20^{\circ}$ & -0.005 & -0.004 & 0.016 & 3.98 & -2.48 & 5.14 \\
\hline & & $40^{\circ}$ & 0.35 & -0.025 & 0.066 & 7.64 & -0.60 & 1.98 \\
\hline
\end{tabular}

packing compared with the parallel packing, whereas a larger increase can be seen for the $Y^{\prime}$-type film. At the moment we are not able to explain this increase in $d_{z z}$ for $Y^{\prime}$-type films.

\section{Refractive index}

The refractive indices are tabulated in Table VI for parallel and herringbone packing. The tables clearly show that it is not possible to distinguish between $X, Y$, and $Z$-type films solely by inspection of the refractive indices. $n_{x}$ and $n_{y}$ increase with tilt for both packings, while $n_{z}$ increases with tilt for the parallel packing, but decreases with tilt for the herringbone packing. In the previous section it was established that the off-diagonal local-field tensor components are responsible for this behavior. Nonzero off-diagonal components (for parallel packing) result in a decrease in $n_{z}$ with tilt, whereas zero off-diagonal components (for herringbone packing) lead to an increase in $n_{z}$ with tilt. $X$-, $Y$-, and $Z$-type films seem to exhibit the same linear response as a result of the nearly equal local-field tensor components. The linear response of $Y^{\prime}$-type films is different from $X$-, $Y$-, and $Z$-type films. This can be attributed to the difference in local-field tensor components.

\section{Quadratic susceptibility}

The results for the quadratic susceptibility for nonuniform response are shown in Table VII for parallel and herringbone packing. $Y$ - and $Y^{\prime}$-type films are centrosymmetric in untilted and parallel tilted packing. This leads to zero quadratic susceptibility components as shown in Table VII. The individual components of successive layers are equal but opposite, thus resulting in zero sum. $X$ - and $Z$-type films are noncentrosymmetric and are equivalent except that their respective $x$ - and $z$-axes are in opposite directions. This is reflected in the results, with the quadratic susceptibilities of $X$ and $Z$-type films differing only in sign. $\chi_{x x x}^{(2)}$ is largest at nearly $52 \mathrm{pm} \mathrm{V}^{-1}$, which is considerably larger than the $x x x$ component of the reduced first hyperpolarizability $(0.75 \mathrm{pm}$ $\mathrm{V}^{-1}$ ). Note, $\chi^{(2)}$ is calculated using Eq. (15) which involves all components of the local field tensor and the reduced first hyperpolarizability.

For herringbone packing, the symmetry of $Y$ - and $Y^{\prime}$-type films reduces to a single mirror plane, and hence the films are NLO-active, as can be seen from Table VII. In this arrangement the mirror plane contains the $x$ - and $y$-axes. The directions of the $z$-axes of successive layers are opposite, with the result that components containing $z$ an odd number of times are zero. These films exhibit large $x x x$ components, 

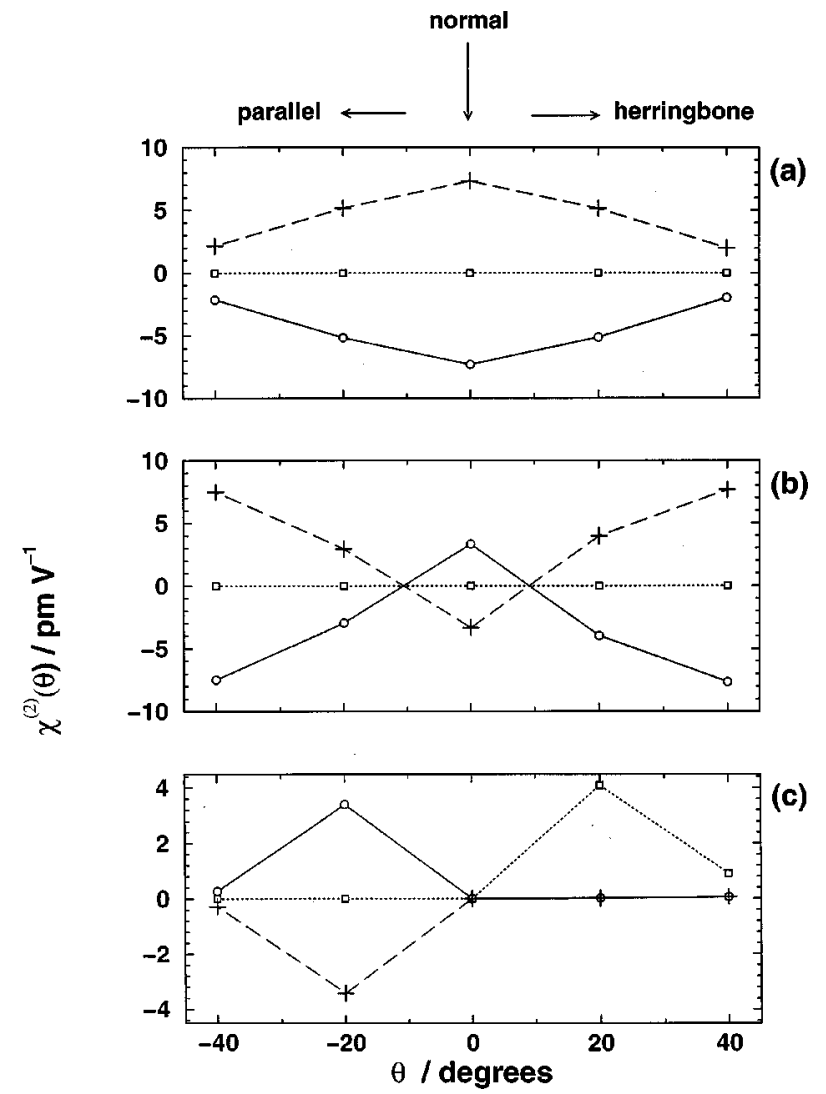

FIG. 4. Variation of quadratic susceptibility with tilt in the $x z$ plane. (a) $z z z$, (b) $z x x$, and (c) $x z z$ components. Three types of Langmuir-Blodgett films with nonuniform distributed polarizability and first hyperpolarizability are compared, $X$-type (solid lines and circles), $Y$-type (dotted lines and squares), and Z-type (dashed lines and plus signs). Normal, parallel, and herringbone refer to different ways of packing in the multilayer unit cell as explained in the text.

nearly $60 \mathrm{pm} \mathrm{V}^{-1}$ for $Y$-type films and approximately $46 \mathrm{pm}$ $\mathrm{V}^{-1}$ for $Y^{\prime}$-type films. The $x z z$ component is very close to zero for $Y^{\prime}$ films, whereas this is about $9 \mathrm{pm} \mathrm{V}^{-1}$ for $Y$-type films. Comparing herringbone packed $X$ - and $Z$-type films, we find that components that contain $x$ an odd number of times are equal both in magnitude and sign, while those that contain $z$ an odd number of times are equal in magnitude but opposite in sign. This can be explained by examining the respective axis directions of the individual layers. Both types of film have opposite $z$-axis directions for successive layers. Hence, the quadratic susceptibility components of the individual layers will be opposite in sign but not in magnitude since the structure is not centrosymmetric. Therefore, the sum of the layer contributions will be nonzero, but small. The $z$-axis direction of successive layers in both $X$ - and $Z$-type films are similar, but differ in sign, and so the quadratic susceptibility components that contain $z$ an odd number of times will be equal in magnitude, but opposite in sign. Figure 4 shows the data for $X-, Y$-, and $Z$-type films in Table VII more clearly. It is obvious that $X$ - and $Z$-type films mirror each other for both types of packing and all angles of tilt for the $z z z$ and $z x x$ components. The $z z z$ components decrease with tilt, whereas the $z x x$ components increase with tilt. The difference between parallel and herringbone packing can be attributed to local-field tensor off-diagonal compo- nents, as already explained in Sec. V B. All displayed components for $Y$-type films are zero except the $x z z$ component which is nonzero for herringbone packing. For herringbone packed $X$ - and $Y$-type films the figure clearly shows that the $x z z$ components are equal in magnitude and sign.

\section{Effect of molecular packing}

The effect of molecular packing is similar to the observations made for films with uniform molecular response. Parallel packed films have nonzero off-diagonal local-field tensor components, whereas herringbone packed films have zero off-diagonal components. Of the other components only the $x x$ components change in magnitude. They increase less with tilt for herringbone packing than for parallel packing. For the refractive index it was found that except for $Y^{\prime}$-type films the $x$ and $y$ components do not change with the packing. $n_{y}$ is larger for the largest angle of tilt for parallel packing, while $n_{z}$ behaves along similar lines as films with uniform molecular response, increasing with ilt for herringbone packing and decreasing for parallel packing. For the quadratic susceptibility the most prominent changes occur in the components that contain $x$ an odd number of times. $Y$ - and $Y^{\prime}$-type films are not centrosymmetric for herringbone packing as opposed to parallel packing and exhibit sizeable components especially for $x x x$. For $X$ - and Z-type films the largest component ( $x x x$ for parallel packing) reduces to nearly zero for herringbone packing. All other components remain independent of the type of packing.

\section{Comparison between uniform and nonuniform distributed response}

A $X$-type film with nonuniform distributed molecular response can be compared with an $X$-type film with uniform distributed molecular response. A similar comparison is possible for $Y$-type films. The conclusions for $X$-type films are also valid for $Z$-type films, since they are equivalent in structure except for the direction of the $z$-axis. Figures 5 and 6 show the local-field tensor and refractive index for $X$-type films with uniform (solid lines and circles) and nonuniform (dotted lines and squares) distributed molecular response, respectively. The information for the quadratic susceptibility can be obtained from Tables IV and VII. The observed trends for packing and tilt for the local-field tensor and refractive are the same for uniform and nonuniform distribution. However, the magnitude of the individual components depends on how the molecular response is distributed. Thus, in general, a uniform distributed polarizability leads to a larger local-field tensor and quadratic susceptibility (except for $x x x$ and $x y y$ ). The refractive indices are larger for nonuniform response, with the exception of $n_{z}$. Comparison between $Y$-type films leads to similar observations.

\section{DISCUSSION AND CONCLUSIONS}

The calculations discussed in this article take us significantly closer towards calculating the optical response of realistic Langmuir-Blodgett films. We have used highly simplified model Langmuir-Blodgett films to gain insight into the difference in optical response for $X-, Y$-, and $Z$-type films. 

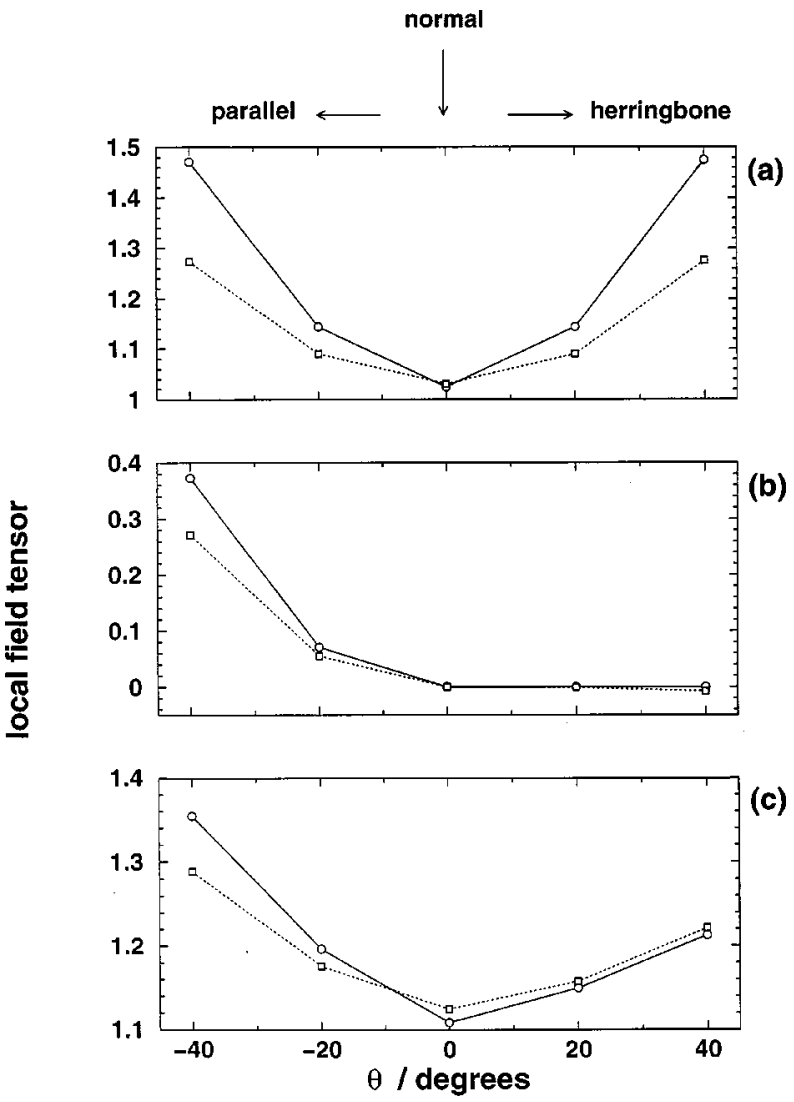

FIG. 5. Comparison between local field tensor for Langmuir-Blodgett films with uniform (solid lines and squares) and nonuniform (dotted lines and squares) distributed molecular response. (a) $z z$, (b) $z x$, and (c) $x x$ components. Normal, parallel, and herringbone refer to different ways of packing in the multilayer unit cell as explained in the text.

The film-forming molecules in these films are usually amphiphilic in nature, i.e., they consist of a hydrophilic head group and a hydrophobic tail. This implies that the molecular response is nonuniformly distributed over the molecule. In order to asses the effect of distributing the molecular response we started our investigations with films with a uniform molecular response. The picture that emerges for tilted films with uniform distributed molecular response is the following: the local-field tensor and refractive index are independent of the type of film, but dependent on the type of molecular arrangement in the multilayer unit cell. The difference between parallel and herringbone packing lies in the distance between the submolecules in adjacent layers. In this type of material under the conditions assumed here, the dominant interaction between molecules is dipolar. Thus, a difference in distance between different types of molecular packing will affect the dipole tensor sum. In turn the dipole tensor sum will affect the local-field tensor. This quantity gives information in which way a material reacts to its environment. We have established that the local-field tensor plays an important role in the trends observed for the refractive index and the quadratic susceptibility. In general it was found that the local-field tensor components increase with the angle of tilt, except for $d_{y y}$. Nonzero off-diagonal components were found only for parallel packing, since herringbone packing has a single mirror plane. The $x$ and $z$ compo-

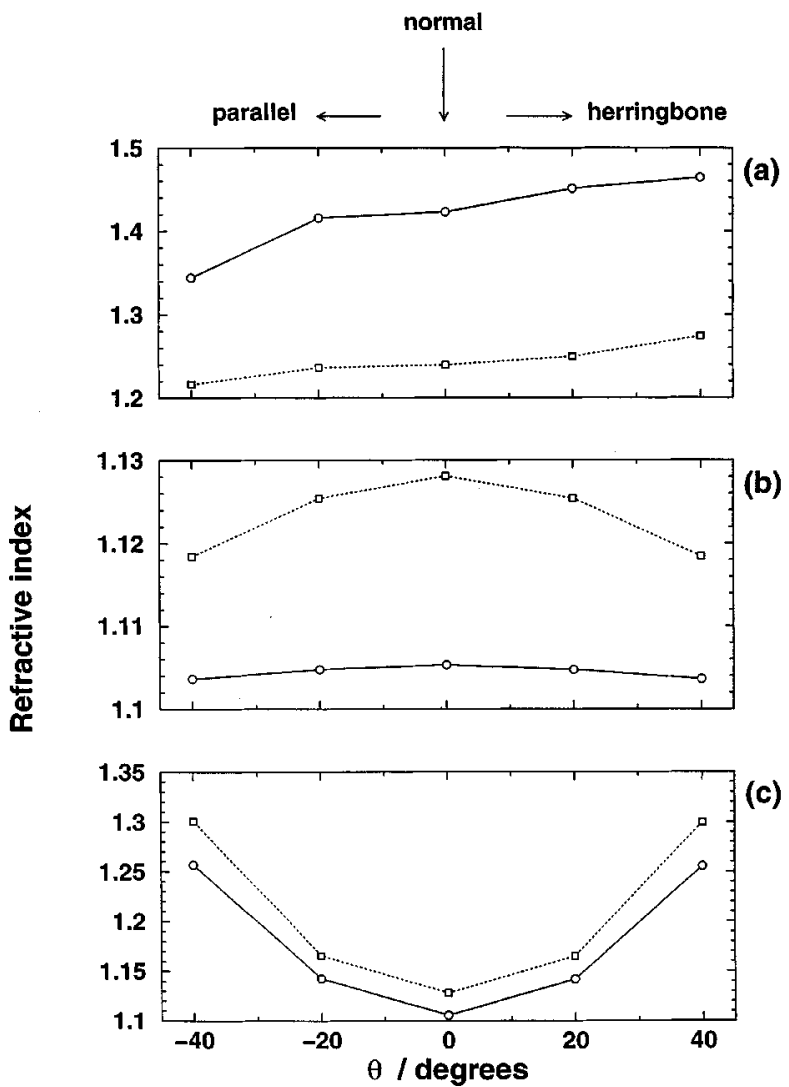

FIG. 6. Comparison between refractive index for Langmuir-Blodgett films with uniform (solid lines and circles) and nonuniform (dotted lines and squares) distributed molecular response. (a) $z$, (b) $y$, and (c) $x$ components. Normal, parallel, and herringbone refer to different ways of packing in the multilayer unit cell as explained in the text.

nents of refractive index increase with tilt for herringbone packing, whereas for parallel packing the $x$ component increases and the $z$ component decreases with tilt. It was established that this behavior is a result of the nonzero offdiagonal local-field tensor components for parallel packing. The quadratic susceptibility depends on both the type of film and its packing. The results reflect the fact that $X_{p}$-type films are noncentrosymmetric, $X_{h}$ and $Y_{h}$ have a mirror plane, and $Y_{p}$ is centrosymmetric. Sizeable components between 15 and $20 \mathrm{pm} \mathrm{V}^{-1}$ for tilted films are found in all type of films, except for $Y_{p}$. For tilted films in which the molecular response is concentrated in the polar heads (of the filmforming molecules) the distance between the heads in adjacent layers affect the results in the following way. The $z z$ component of the local-field tensor varies inversely with the distance in the $z$ direction between the heads in adjacent layers, whereas the $x x$ component varies directly with the distance between the heads in the $x$ direction. The influence of the type of packing on the results is similar to that observed for films with uniform molecular response. Here, we discuss the effect of the changes in the distance between the head and tail groups in adjacent layers. In fact, the difference in the magnitude of the local-field tensor components between both types of packings is a result of head-tail interactions. The local-field tensor components can be ordered with respect to their head-head distance. The head-head dis- 
tances increases from $Y^{\prime}$ through $X$ and $Z$ until the largest distance is found in $Y$-type films. The refractive indices follow this pattern, allowing for the effect of the off-diagonal local-field tensor components. Similar observations can be made for the quadratic susceptibility components. In films with nonuniform molecular response it was expected that the optical response of $Y$-type films would be enhanced if the heads were placed adjacent, as opposed to being furthest apart ( $Y^{\prime}$-type). Our calculations show that this is valid only for the $z x$ and $z z$ components of the local-field tensor and the $z$ component of the refractive index. For all other components and all nonzero components, the response of $Y$-type films is larger than the response of $Y^{\prime}$-type films.

A question worth asking is whether conventional Langmuir-Blodgett films consisting of surfactant molecules with polar head are indeed the best building blocks for creating optical active materials. These molecules have a nonuniform distributed molecular response. But what about molecules with a more uniform distributed molecular response? Block copolymers are a suitable example; they are interfacial active and thus able to form monolayers, which are necessary for constructing Langmuir-Blodgett films. Films consisting of molecules with uniform and nonuniform distributed response were compared in Sec. V D. However, the results do not present us with a straightforward choice.
In a subsequent article, ${ }^{14}$ we address substrate effects on the linear and quadratic optical response in model Langmuir-Blodgett multilayers.

\section{ACKNOWLEDGMENTS}

We thank M. Malagoli for useful discussions. M.i.h.P. acknowledges support from the EU TMR Network Programme under Contract No. FMRX-CT96-0047.

${ }^{1}$ M. R. Philpott, J. Chem. Phys. 61, 5306 (1974).

${ }^{2}$ F. W. de Wette and G. E. Schacher, Phys. Rev. 137, A37 (1965).

${ }^{3}$ M. R. Philpott, J. Chem. Phys. 58, 588 (1973).

${ }^{4}$ R. W. Munn and M. M. Shabat, J. Chem. Phys. 99, 10052 (1993).

${ }^{5}$ R. W. Munn and M. M. Shabat, J. Chem. Phys. 99, 10059 (1993).

${ }^{6}$ R. W. Munn, J. Mater. Chem. 4, 849 (1994).

${ }^{7}$ L. Bourdieu, P. Silberzan, and D. Chatenay, Phys. Rev. Lett. 67, 2029 (1991)

${ }^{8}$ E. Meyer, L. Howald, R. M. Overney, H. Heinzelman, J. Frommer, H.-J. Güntherodt, T. Wagner, H. Schier, and S. Roth, Nature (London) 349, 398 (1991).

${ }^{9}$ A. Baggaley, M. Bishop, J. H. R. Clarke, L. E. Davis, T. A. King, D. A. Leigh, F. R. Mayers, A. Mohebati, R. W. Munn, M. M. Shabat, O. Szczur, D. West, and J. O. Williams, Mol. Cryst. Liq. Cryst. 235, 201 (1993)

${ }^{10}$ D. R. Swanson, T. Luty, and C. J. Eckhardt, J. Chem. Phys. 107, 4744 (1997).

${ }^{11}$ T. Luty, D. R. Swanson, and C. J. Eckhardt, J. Chem. Phys. 110, 2606 (1999).

${ }^{12}$ R. W. Munn, J. Chem. Phys. 97, 4532 (1992).

${ }^{13}$ R. W. Munn, J. Chem. Phys. 101, 8162 (1994).

${ }^{14} \mathrm{M}$. in het Panhuis and R. W. Munn (unpublished). 